\title{
El Comité Asesor Internacional en el año 2014
}

\author{
HUMBERTO REYES B. ${ }^{1}$, MAX ANDRESEN H. ${ }^{2}$, JOAQUÍN PALMA H. ${ }^{2}$
}

\section{The International Advisory Committee in the year 2014}

In 2001 an International Advisory Committee was instituted in Revista Médica de Chile, including in it prominent physicians working out of Chile who maintain regular contacts with different Chilean societies in the fields of Internal Medicine and its subspecialties. Members in this Committee are expected to contribute with their advice in the contents of our journal and also as expert reviewers and writers. Regrettably, Richard V. Lee, MD, passed away in May 2013. Vincenza Snow, MD, changed her professional activities and had to leave the Committee. Two new members are incorporated since January 2014: Juan Dapueto, MD, Professor of Psychiatry and Internal Medicine from Montevideo, Uruguay, with particular interest in quality of life in patients with chronic diseases, and Diego Gracia, MD, Professor of Psychiatry from Madrid, Spain, a distinguished scholar in the fields of History of Medicine and Bioethics.

(Rev Med Chile 2014; 142: 7-8)

Key words: Journalism, medical; Peer review; Periodicals; Writing.

E International Advisory Committee, o Comité Asesor Internacional, fue establecido en esta revista en el año $2001^{1}$. Lo han integrado prominentes médicos, científicos y docentes de países de Europa y Norteamérica, conectados con el ambiente de la medicina en nuestro país al participar en congresos, simposios y otras reuniones científicas. Cinco de ellos se habían graduado como médicos en Chile y después emigraron a otros países donde han desarrollado su carrera académica: las Dras. Marcela Contreras y Vincenza Snow, y los Dres. Felipe Cabello, Jorge Rakela y Moisés Selman ${ }^{2}$. Han destacado por la frecuencia de sus contribuciones con manuscritos destinados a la Revista los Dres. Felipe Cabello, Jorge Rakela y Richard V. Lee; otros miembros han participado en la revisión de manuscritos.

En el año 2012 terminó la participación de la Dra. Vincenza Snow, quien dejó de ser funcionaria del American College of Physicians y no podía seguir colaborando en este comité. En mayo de 2013 debimos lamentar el deceso del Dr. Richard V. Lee, cuya notable participación en el XXXI Congreso Mundial de Medicina Interna, en Santiago, comprometió la gratitud de sus organizadores y de los asistentes.

En enero de 2014 damos la bienvenida a dos nuevos miembros, seleccionados con criterios similares a sus predecesores: El Dr. Juan Dapueto P. es un distinguido académico uruguayo que combina las especialidades de psiquiatría y medicina interna para estudiar y resolver problemas relacionados con calidad de vida, particularmente en pacientes con enfermedades renales crónicas. El Dr. Diego Gracia G., psiquiatra español de renombre internacional en historia de la medicina y ética médica, ha sido profesor invitado en varios cursos y conferencias en nuestro país, y es Académico Honorario en la Academia Chilena de Medicina.

Al presentar a estos nuevos miembros del Comité Asesor Internacional, agregando sus fotografías y una síntesis de sus antecedentes curriculares, les expresamos nuestros deseos de una fértil colaboración.

\section{Referencias}

1. Reyes H, Kauffmann R, Andresen M. El Comité Asesor Internacional (Editorial). Rev Med Chile 2001; 128: 1349-53.

2. Reyes H, Palma J, Andresen M. El Comité Asesor Internacional desde el año 2009 (Editorial). Rev Med Chile 2009; 137: 593-8. 


\section{Nuevos miembros del Comité Asesor Internacional}

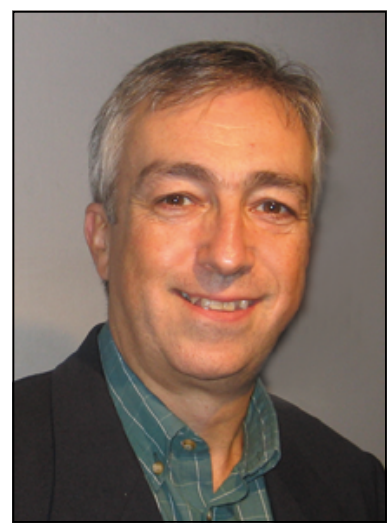

\section{Dr. Juan José Dapueto Prieto}

Nació en Montevideo, Uruguay. En la Universidad de la República, Uruguay, obtuvo títulos de Doctor en Medicina, Especialista en Psiquiatría de adultos y Especialista en Medicina Interna. Tiene el grado de Doctor en Ciencias Biomédicas por el Programa de Investigaciones Biomédicas de la Escuela de Graduados, Facultad de Medicina, Fundación Manuel Pérez, Universidad de la República, Uruguay.

Es Director del Departamento Clínico de Medicina del Hospital de Clínicas y Director del Departamento de Psicología Médica de la Facultad de Medicina. Integrante del Sistema Nacional de Investigadores de la Agencia Nacional de Innovación e Investigación, en Uruguay.

Es Editor Asociado de la revista Quality of Life Research, Journal of the International Society for Quality of Life Research, Miembro del Editorial Board de Nephrology, Dialysis and Transplantation, Journal of the European Nephrology Association; y revisor de artículos para Quality of Life Research; Journal of Psychosomatic Research; Psycho Oncology, Health and Quality of Life Outcomes; Journal of Clinical Epidemiology; Archivos de Pediatría del Uruguay, Revista Médica del Uruguay. Es autor y coautor de artículos sobre calidad de vida y otros temas, en revistas médicas internacionales, uruguayas y chilenas.

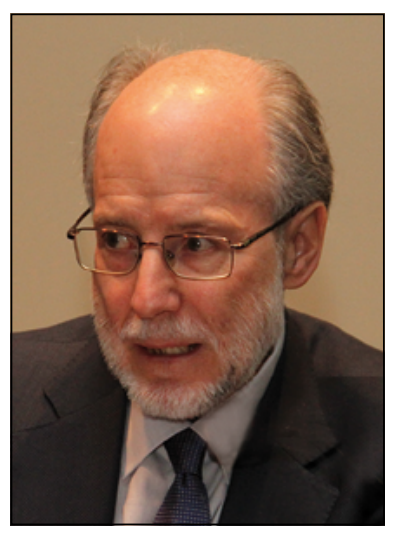

\section{Dr. Diego Gracia Guillén}

Nació en Madrid, España, el 21 de mayo de 1941. Licenciado en Medicina en 1970 por la Universidad de Salamanca con Premio Extraordinario. Alumno Interno por oposición de la Cátedra de Psiquiatría. Doctor en 1973 por la Universidad Complutense. Diplomado en Psicología Clínica por la Universidad Pontificia de Salamanca. Especialista en Psiquiatría. Amplió estudios en Nassau, Heidelberg, París y en varios centros de Estados Unidos. Colaborador científico del CSIC, por oposición, en 1974. Profesor agregado de Historia de la Medicina (Antropología Médica) en 1978 y desde 1979 Catedrático de Historia de la Medicina de la Universidad Complutense (UCM). Fue vicedecano de la misma Facultad desde 1981 hasta 1983. Ha sido director de la biblioteca de esta Facultad hasta el año 2003. Director del Magíster en Bioética de la UCM. Director de la Fundación Xavier Zubiri. Presidente del Patronato de la Fundación de Ciencias de la Salud y director de su Instituto de Bioética. Ha publicado, entre otros, los libros: "Teología y medicina en la obra de Miguel Servet" (1981), "Ética de la calidad de vida" (1984), "Voluntad de verdad. Para leer a Zubiri" (1986), "Historia del medicamento" (1987), "Fundamentos de bioética" (1989), "Primum non nocere: El principio de no maleficencia como fundamento de la ética médica" (1990), "Procedimientos de decisión en ética clínica" (1991), "Etica y vida: Estudios de bioética” (4 vols, 1998), "Medice, cura te ipsum: Sobre la salud física y mental de los profesionales sanitarios" (2004), "Como arqueros al blanco: Estudios de bioética" (2004).

El Profesor Gracia fue discípulo del Profesor Pedro Laín Entralgo y se le considera su sucesor en los campos de la Historia de la Medicina y la Bioética. Ha participado como Profesor Invitado en cursos de Bioética en nuestro país y desde el año 2001 es Académico Honorario Extranjero en la Academia Chilena de Medicina, del Instituto de Chile. 